\title{
Optimisation de la mesure urinaire des émetteurs bêta par scintillation liquide à résolution temporelle
}

\author{
G. Finance ${ }^{a}$, J. Siscard, M. Agarande, D. Franck et P. Boisson \\ IRSN, PRP-HOM, Service de Dosimétrie Interne, BP 17, 92262 Fontenay-aux-Roses Cedex, France.
}

Reçu le 24 juillet 2012 - Accepté le 18 octobre 2013

\begin{abstract}
Résumé - La scintillation liquide est une méthode d'analyse de choix du suivi radio toxicologique des travailleurs susceptibles d'être exposés à des sources émettrices $\beta$ purs. Les éléments étant présents à l'état de traces dans les urines, il est nécessaire d'optimiser la technique de mesure afin d'obtenir la meilleure sensibilité. Ces dernières années ont vu apparaître des nouvelles générations de liquides scintillants, moins polluants et moins toxiques, destinés à remplacer les liquides scintillants traditionnels. Ces scintillants dits de «nouvelle génération » possèdent cependant un inconvénient technique à leur utilisation : une dissipation plus longue de l'énergie émise par le photon de fluorescence résultant de l'interaction entre le milieu scintillant et le rayonnement. Ce phénomène a un impact direct sur la qualité de la détection et amène à rechercher une valeur du Delay Before Burst optimale qui permette de distinguer les signaux du rayonnement de la source à mesurer, de ceux venant du milieu environnant. Cette étude vise à montrer l'importance du choix de la valeur du paramètre Delay Before Burst dans l'optimisation des limites de détection pour les mesures en scintillation liquide. L'influence de ce paramètre a été étudiée sur les différents radionucléides mesurés au Laboratoire d'Analyses Médicales Radiotoxicologiques (LAMR) de l'Institut de Radioprotection et de Sureté Nucléaire (IRSN); le ${ }^{3} \mathrm{H}$, le ${ }^{14} \mathrm{C}$ et le ${ }^{89} \mathrm{Sr}$. Notre étude compare les rayonnements $\beta$ mesurés avec un liquide scintillant traditionnel : le Pico-Fluor 40 et un liquide scintillant de nouvelle génération : l'Ultima Gold LTT.
\end{abstract}

\begin{abstract}
Optimization of the measurement of urinary beta transmitters by scintillation liquid with delay before burst. In a follow-up of radiotoxicological staff working with $\beta$-emitting sources through bioassays, liquid scintillation is the best method to achieve this type of analysis. As the elements are present in trace amounts in urine, it is important to optimize the methods to achieve the best sensitivity. In recent years, less polluting and less toxic scintillating liquids have become available and should eventually replace the current ones. Besides these new features, they take longer to dissipate the energy emitted by the fluorescence photon resulting from the interaction between the $\beta$-particle and the scintillators. This has a direct impact on the quality of the detection and requires setting optimal detection parameters; in particular, the Delay Before Burst must be carefully tuned. This study aims at showing the importance of tuning the Delay Before Burst parameter to optimize the detection limits in liquid scintillation. It covers the whole range of energies of $\beta$ radiation measured $\left({ }^{3} \mathrm{H},{ }^{14} \mathrm{C}\right.$ and $\left.{ }^{89} \mathrm{Sr}\right)$ and two types of scintillating liquid used in the laboratory: one current type, the Pico-Fluor 40, and a new one, the Ultima Gold LTT.
\end{abstract}

Keywords: liquid scintillation / Delay Before Burst / beta emission $/{ }^{3} \mathrm{H} /{ }^{14} \mathrm{C} /{ }^{89} \mathrm{Sr} /$ urine radiotoxicology

\section{Introduction}

En cas de contamination interne, la radio toxicologie urinaire est l'une des méthodes à mettre en œuvre pour qualifier et quantifier les radionucléides incorporés. Quand la contamination interne est imputable à des radionucléides émetteurs $\beta$, la mesure par scintillation liquide reste une méthode de choix (Simonnet, 1994).

Le principe en est aujourd'hui bien connu. Lorsque la particule $\beta$ traverse le milieu détecteur, ici le liquide scintillant, elle interagit en provoquant l'excitation des molécules fluorescentes (Horrocks, 1974), qui lors de leur retour à l'état

\footnotetext{
a gregory.finance@irsn.fr
}

fondamental vont émettre à leur tour des photons. Ces photons seront détectés par l'intermédiaire de deux photomultiplicateurs, généralement montés en coïncidence, et associés à une électronique aboutissant au final à l'émission d'un signal électrique (Cassette, 2004).

Ce dispositif n'est cependant pas suffisant pour discriminer complètement le signal d'une source à mesurer de celui originaire d'un mouvement propre. Pour mieux les différencier, certains appareils de comptage ont été équipés d'une fonction de détection du mouvement propre se faisant par la reconnaissance du spectre temporel : la fonction «Delay Before Burst » (DBB). Ainsi en observant la hauteur de l'impulsion en fonction de l'énergie et en y associant l'échelle du temps, il est 


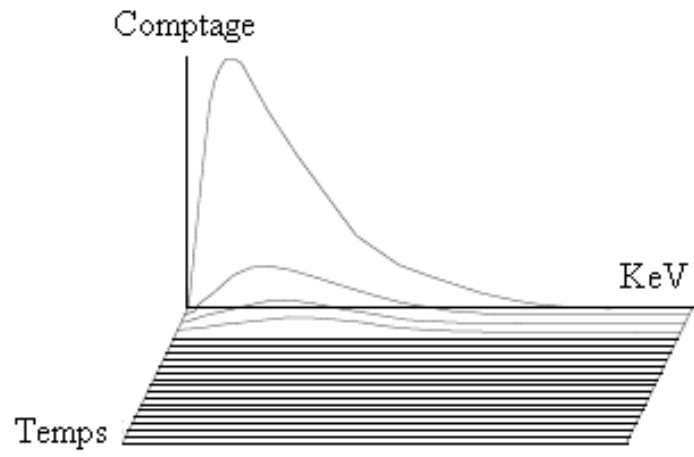

Fig. 1. Schéma en 3D d'un spectre d'une impulsion bêta. $3 \mathrm{D}$ representation of a beta pulse spectrum.

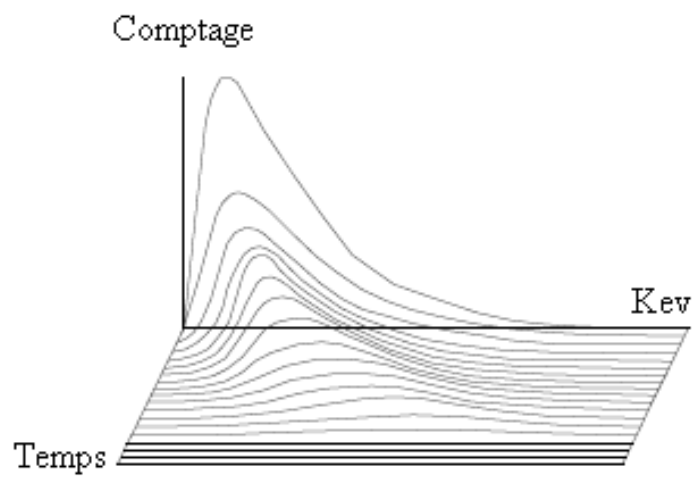

Fig. 2. Schéma en 3D d'un spectre d'une impulsion Mouvement propre.

3D representation of a background pulse spectrum.

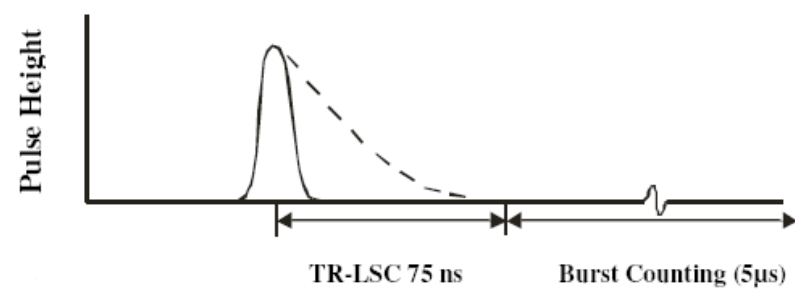

Fig. 3. Principe d'un détecteur à scintillation liquide à temps fixe avant comptage des post impulsions.

Principle of a liquid scintillation detector with fixed time intervals before detection of the afterpulses.

possible de différencier le signal émis par le radionucléide présent dans la préparation, un pic suivi d'une «traine» (Fig. 1), de celui issu du mouvement propre, un pic suivi d'une série de post-impulsions (Fig. 2).

Comment dissocier cette «traine » associée au radionucléide à mesurer, des post-impulsions issues du mouvement propre? C'est la question qui s'est posée pour vérifier l'impact sur les performances des mesures.

Une première génération d'appareils a évolué en s'équipant d'une fonction DBB à temps fixe de 75 ns. Le principe étant qu'au-delà de ce temps, le signal est identifié comme relevant d'une post-impulsion associée au mouvement propre (Fig. 3). Lorsque le signal et sa traine apparaissent dans des délais inférieurs à 75 ns, ils sont identifiés comme provenant

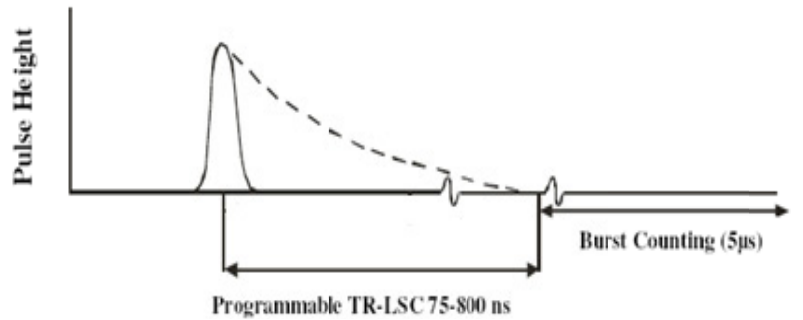

Fig. 4. Principe d'un détecteur à scintillation liquide à temps réglable avant comptage des post impulsions.

Principle of a liquid scintillation detector with adjustable time intervals before detection of the afterpulses.

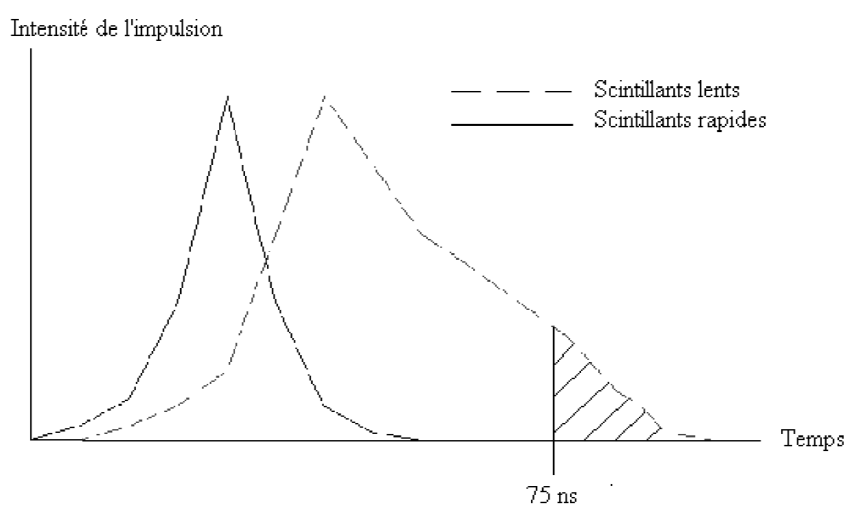

Fig. 5. Différence de comportement entre un liquide scintillant dit « rapide » et un liquide scintillant dit «lent».

Difference between so-called "fast" and "slow" scintillation fluids.

du radionucléide à mesurer. Il convient de souligner que si la «traine » se propage au-delà de ce délai, elle est reconnue comme post-impulsion et le signal est identifié en tant que mouvement propre.

Le principe d'une fonction DBB à temps fixe fonctionnait correctement avec les liquides scintillants traditionnels (dits « rapides ») les photons se dissipaient rapidement et la «traine» ne s'étendait pas au-delà de 75 ns (Fig. 3). À l'arrivée des liquides scintillants de nouvelle génération (dits «lents » par opposition), le problème de la durée de la «traine » du signal s'est à nouveau posé : la «traine » associée au signal pouvait s'étendre au-delà de 75 ns. L'Ultima gold LLT, réputé plus performant pour la mesure des émetteurs de faible énergie, et moins polluant, en est un exemple (Perkin Elmer, 2007).

La fonction DBB a dû être encore améliorée et est devenue paramétrable pour mieux prendre en compte les spécificités des liquides scintillants de différentes générations et les différents types de signaux : le temps fixé à 75 ns jusqu'alors par défaut est devenu ajustable jusqu'à 800 ns (Figs. 4 et 5) (Passo et al., 1992).

Afin de mettre en évidence cette différence de comportement et d'optimiser les méthodes de mesure, le laboratoire a entrepris une étude sur plusieurs types de liquide scintillant. Différents radionucléides émetteur $\beta$ ont été mesurés couvrant toute la gamme énergétique des éléments habituellement mesurés au laboratoire. 


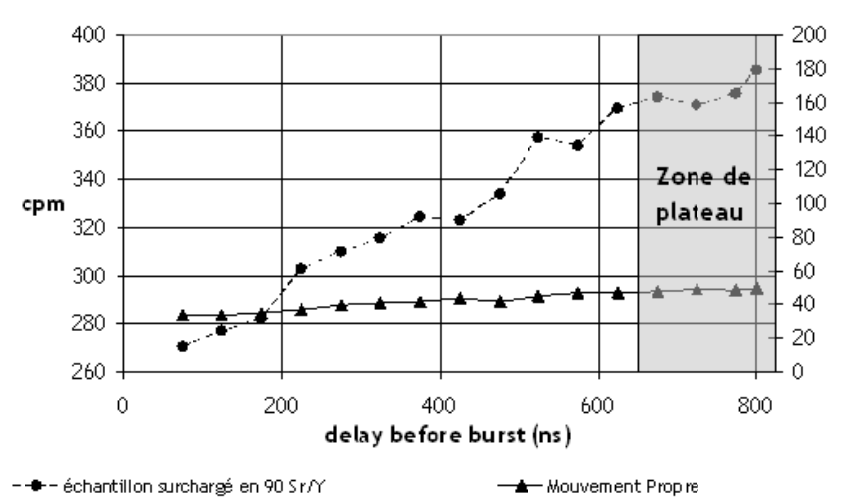

Fig. 6. Variation du nombre de cpm en fonction du DBB en utilisant comme liquide scintillant l'Ultima Gold LLT et ce pour la mesure $\mathrm{du}{ }^{90} \mathrm{Sr} /{ }^{90} \mathrm{Y}$.

Evolution in number of cpm as a function of the DBB and detection of ${ }^{90} \mathrm{Sr} /{ }^{90} \mathrm{Y}$ in the fluid Ultima Gold LLT.

\section{Matériels et méthodes}

Différentes séries d'échantillon contenant du ${ }^{3} \mathrm{H}$, du ${ }^{14} \mathrm{C}$ et du ${ }^{89} \mathrm{Sr}$ ont été préparées pour mettre en évidence l'influence du réglage du DBB sur leur efficacité de comptage et par conséquent leurs limites de détection. Un réglage commun à l'ensemble des séries a été déterminé. Les échantillons ont été préparés, pour de la mesure directe (Tymen et al., 1998), selon le protocole appliqué au laboratoire (LAMR/MOP-001) : un prélèvement d'urine $(2 \mathrm{ml})$ surchargé en radionucléide d'activité connue à valeur assignée auquel est ajouté une quantité fixe de liquide scintillant $(18 \mathrm{ml})$. Le temps de comptage est fixé à une heure.

Deux liquides scintillants ont été utilisés pour les besoins de l'étude :

- un liquide scintillant traditionnel, dit « rapide» : le PicoFluor 40,

- un liquide scintillant de nouvelle génération, récent, dit « lent » : l'Ultima Gold LLT. Ce scintillant présente l'avantage d'être biodégradable et permet de faciliter la gestion des déchets de ce type.

Les différents échantillons ont été mesurés sur un même appareil, un compteur à scintillation liquide de type TRI-CARB 3100 TR de Perkin Elmer. Cet appareil est équipé d'une fonction proposant une plage de DBB ajustable, comprise entre $75 \mathrm{~ns}$ et $800 \mathrm{~ns}$. Les mesures ont été réalisées sur cette plage en faisant varier la fonction par pas de $50 \mathrm{~ns}$. Les comptages ont été réalisés sur la totalité de la fenêtre, de 0 à $2000 \mathrm{keV}$, en mode normal.

La valeur optimale du DBB est déterminée graphiquement. Sur le graphe présenté sur la figure 6, les impulsions liées à l'échantillon sont matérialisées par la courbe en pointillés avec lecture sur l'échelle de gauche. Les impulsions liées au mouvement propre, le blanc réalisé avec de l'urine et du liquide scintillant, sont matérialisées par la courbe en trait plein et la lecture se fait sur l'échelle de droite. La valeur du DBB est optimale lorsqu'un palier est atteint (Fig. 6). Cette valeur est ensuite confirmée par le calcul via l'estimation du facteur de mérite (valeur du rapport signal/mouvement propre) qui est maximum pour le réglage optimum :

$$
\mathrm{FM}=\mathrm{E}^{2} / \mathrm{B}
$$

avec FM : facteur de mérite, E : efficacité de comptage (0 à 1), B : mouvement propre (impulsion).

Les efficacités de mesure sont calculées en fonction des gammes de quenching du laboratoire et propres à chaque radionucléide mesuré et à chaque appareil. Elles sont exprimées sous la forme :

$$
\mathrm{E}=\mathrm{a}(\mathrm{tSIE})^{3}+\mathrm{b}(\mathrm{tSIE})^{2}+\mathrm{c}(\mathrm{tSIE})+\mathrm{d}
$$

E : efficacité de comptage,

$\mathrm{a}, \mathrm{b}, \mathrm{c}, \mathrm{d}$ : coefficients du polynôme,

tSIE : transformed Spectral Index of External Standard.

Le laboratoire doit disposer d'un protocole de mesure unique et déterminer un DBB commun servant à la détection de tous les radionucléides à mesurer. Pour ce faire, l'influence du DBB a été comparée sur deux temps extrêmes : le premier à 75 ns correspondant au seuil minimal de possibilité de réglage et le second à $675 \mathrm{~ns}$, correspondant à l'entrée du plateau pour le couple ${ }^{90} \mathrm{Sr} /{ }^{90} \mathrm{Y}$ (radionucléide le plus énergétique) qui présente une grande sensibilité à ce paramètre lorsqu' on le mesure avec l'Ultima Gold LLT (Fig. 6).

Les limites de détection (LD) sont déterminées pour chaque radionucléide en fonction des deux valeurs de DBB retenues (75 et $675 \mathrm{~ns}$ ), le calcul est fondé sur la méthode du Groupe Technique de Normalisation $n^{\circ} 5$ (G.T.N.5) utilisée en spectrométrie gamma.

$$
\mathrm{LD}=\frac{4\left[1+\sqrt{1+\left(2\left(\mathrm{MP}+\mathrm{N}_{\mathrm{K}}\right)\right)}\right] \times 1000}{\mathrm{tEPeC}}(\mathrm{Bq} / \mathrm{L})
$$

avec

MP : nombre d'impulsions associées au mouvement propre (impulsions),

$\mathrm{N}_{\mathrm{K}}$ : estimation de la contribution $d \mathrm{du}^{40} \mathrm{~K}$ dans la fenêtre $0 \rightarrow$ Emax du radioélément étudié (impulsion),

$\mathrm{t}$ : temps de comptage (seconde),

E : efficacité de comptage déterminé par la courbe de quenching (0 à 1),

$\mathrm{C}$ : coefficient de décroissance du radionucléide mesuré (0 à 1$)$,

Pe : prise d'essai de l'échantillon urinaire (litre).

La présence de ${ }^{40} \mathrm{~K}$ (émetteur $\beta$ ) dans les urines est quasi systématique. Il est donc un interférant variable qu'il faut prendre en compte dans l'expression des limites de détection. Afin de s'affranchir de la variabilité de ce paramètre dans notre étude, nous avons travaillé sur un pool urinaire unique.

Afin de visualiser l'influence du DBB sur le résultat final, les activités mesurées à 75 ns et à 675 ns ont été comparées aux activités cibles avec un test d'écart normalisé En :

$$
\mathrm{En}=\left|\frac{(\mathrm{Ac}-\mathrm{Am})}{\sqrt{\left((\mathrm{UAc})^{2}+(\mathrm{UAm})^{2}\right)}}\right|
$$

avec

Ac : activité cible $(\mathrm{Bq} / \mathrm{L})$,

Am : activité mesurée $(\mathrm{Bq} / \mathrm{L})$,

UAc : incertitude sur l'activité cible à $\mathrm{k}=2(\mathrm{~Bq} / \mathrm{L})$,

UAm : incertitude sur l'activité mesurée à $\mathrm{k}=2(\mathrm{~Bq} / \mathrm{L})$. 
Tableau 1. Limites de détection et facteurs de mérite. Detection limits and figures of merit.

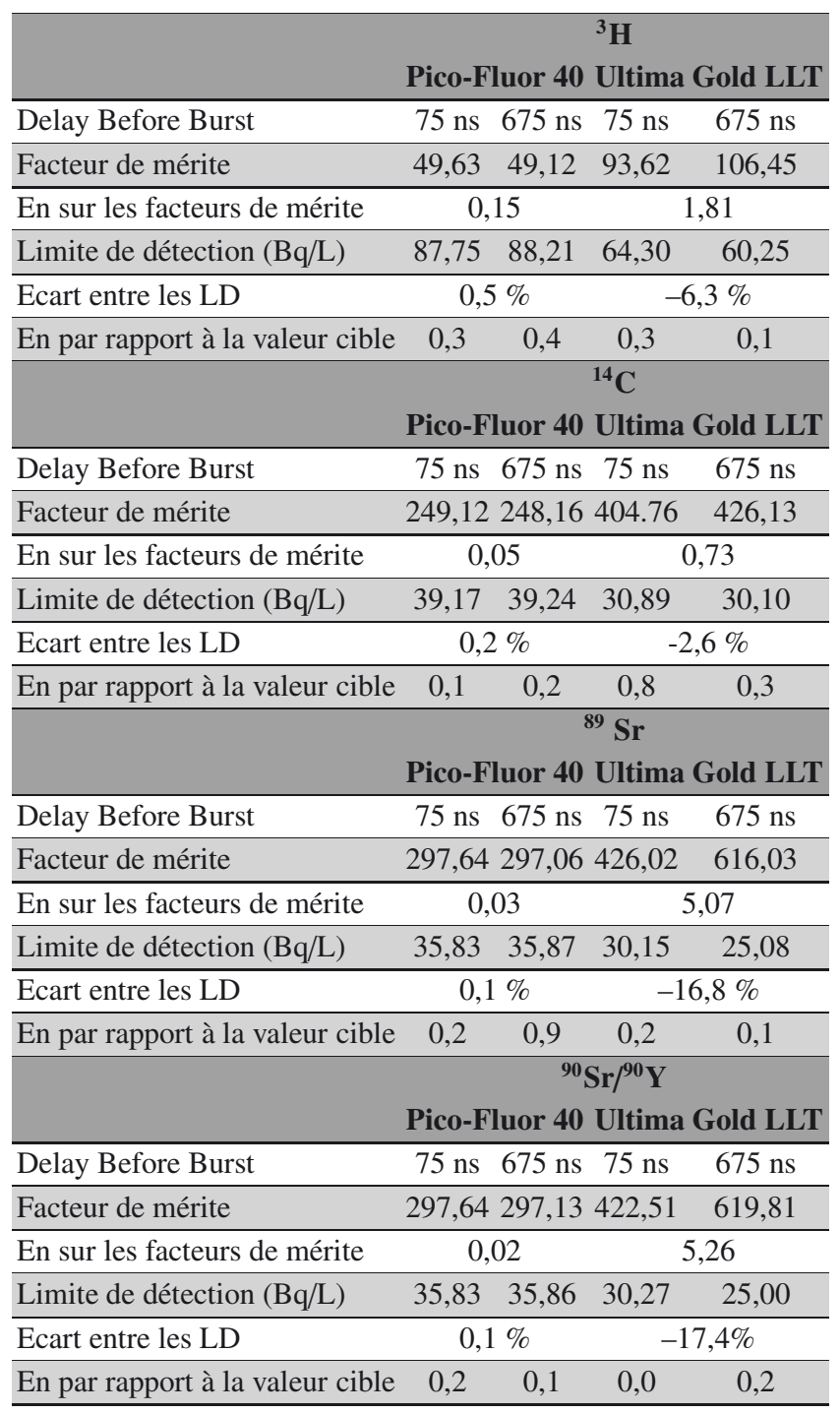

\section{Résultats}

\subsection{Influence du Pico-Fluor et de l'Ultima Gold LLT sur le DBB}

\subsubsection{Cas du ${ }^{3} \mathrm{H}(\operatorname{Emax}=18,56 \mathrm{kev})$}

Sur les préparations réalisées avec du Pico-Fluor 40, il n'est pas constaté de différence significative quelle que soit la valeur du DBB (Tab. 1). Le signal mesuré provenant de la source n'indique pas de hausse sensible (nombre de cpm inchangé), et il en est de même pour la préparation correspondant à un blanc, ce qui se traduit par un facteur de mérite identique. L'efficacité de comptage calculée est la même pour les deux valeurs de délai étudiées (Fig. 8) et les limites de détection obtenues sont équivalentes (Fig. 9). Lorsque l'on compare les valeurs d'activités mesurées, il n'est pas observé de différence significative entre celles calculées à $75 \mathrm{~ns}$ et celle à $675 \mathrm{~ns}$; le test En par rapport à la valeur assignée donne respectivement

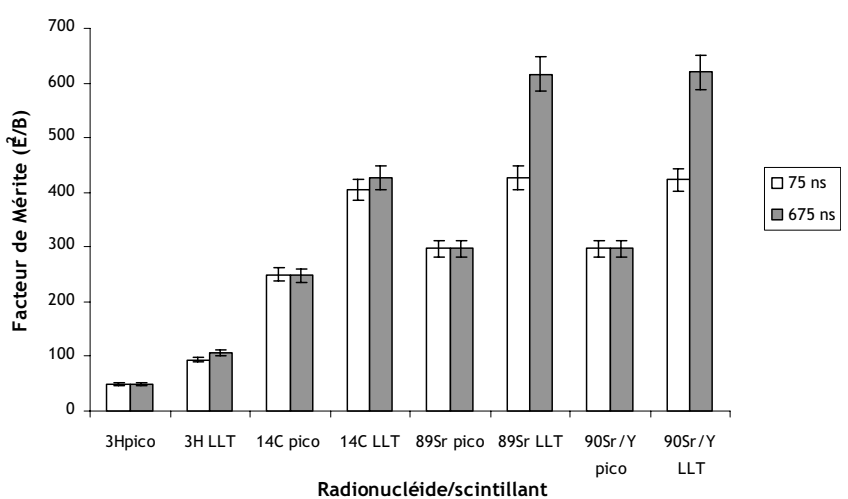

Fig. 7. Variation du facteur de mérite en fonction du couple radionucléide / liquide scintillant.

Variation of the figure of merit based on the couple radionuclide / scintillation fluid.

des valeurs de 0,3 et 0,4 (Tab. 1 ) ce qui démontre que le DBB n'a pas d'influence sur le résultat d'activité rendu. L'optimisation du DBB ne présente pas d'intérêt dans ce cas-là. En revanche, pour les préparations réalisées avec de l'Ultima Gold LLT, l'efficacité de comptage calculée est légèrement améliorée lorsque le DBB est fixé à 675 ns (Fig. 8). Une diminution de $6 \%$ est observée sur les limites de détection. En comparant les facteurs de mérite à 75 ns et à 675 ns grâce au test En (Tab. 1), on observe une différence significative. En effet le En calculé est supérieur à 1,2, montrant que l'optimisation du DBB a un impact non négligeable sur le rapport efficacité/bruit de fond. Lorsque l'on compare les valeurs d'activités mesurées, bien que plus proches de la valeur cible dans le second cas, il n'est pas observé de différence significative entre celles calculées à 75 ns et celles à 675 ns ; le test En par rapport à la valeur assignée donne respectivement des valeurs de 0,3 et 0,1 (Tab. 1), démontrant que quelle que soit la valeur du DBB le résultat d'activité rendu reste juste. Dans ce cas de préparations avec l'Ultima Gold LTT, il est donc important que ce réglage soit optimisé car l'impact sur le résultat global est visible.

\subsubsection{Cas du ${ }^{14} \mathrm{C}(\mathrm{Emax}=156,47 \mathrm{kev})$}

Concernant les préparations réalisées avec du PicoFluor 40, il n'est pas constaté de différence significative quelle que soit la valeur du DBB (Tab. 1). Le rapport efficacité sur bruit de fond reste le même (Fig. 7). L'efficacité de comptage calculée est la même pour les deux valeurs de délai étudiées, le facteur de mérite n'est pas différent et les limites de détection atteintes sont équivalentes (Fig. 9). Lorsque l'on compare les valeurs d'activités mesurées, il n'est pas observé de différences significatives entre celles calculées à 75 ns et celles à 675 ns; le test En par rapport à la valeur assignée donnant respectivement des valeurs de 0,1 et 0,2 (Tab. 1) ce qui démontre que le DBB n'a pas d'influence sur le résultat d'activité rendu. L'optimisation du DBB ne présente pas d'intérêt dans ce cas-là. Comme dans le cas du ${ }^{3} \mathrm{H}$, les préparations réalisées avec de l'Ultima Gold LLT voient leurs efficacités de comptage calculées s'améliorer lorsque le DBB est fixé à 


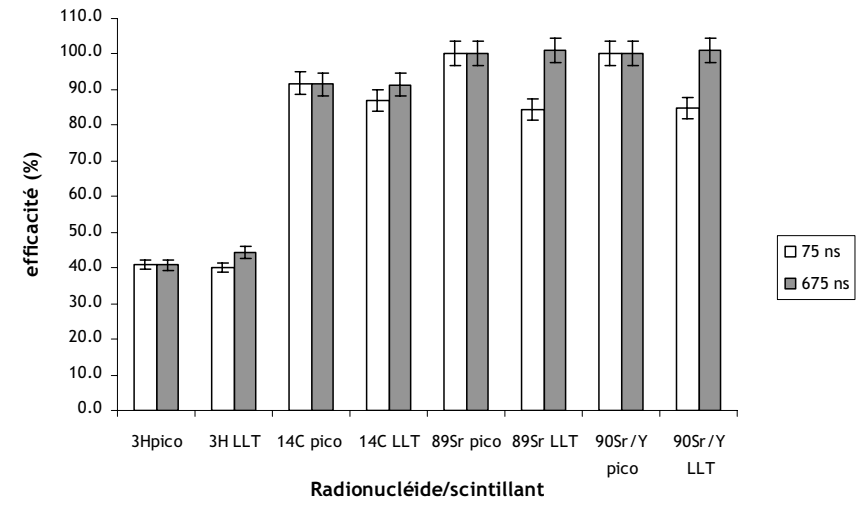

Fig. 8. Variation de l'efficacité de comptage en fonction du couple radionucléide / liquide scintillant.

Variation of the counting efficiency based on the couple radionuclide / scintillation fluid.

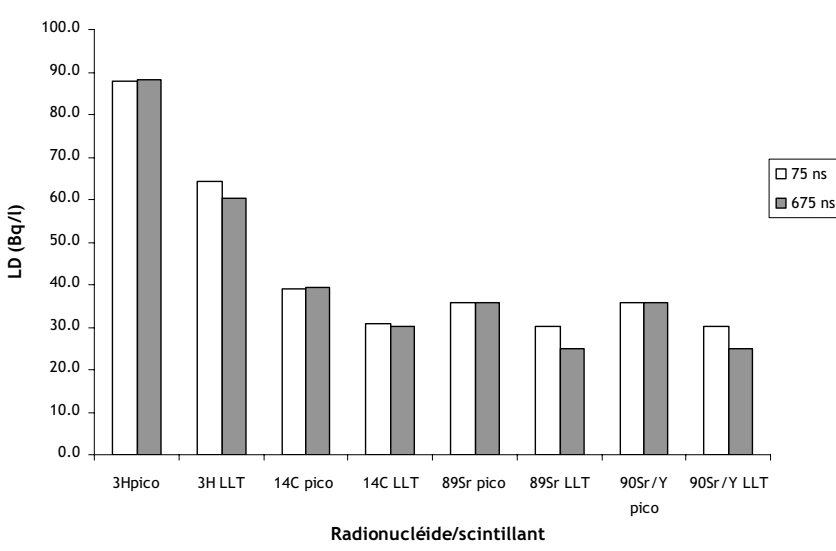

Fig. 9. Variation de la limite de détection en fonction du couple radionucléide / liquide scintillant.

Variation of the detection limit based on the couple radionuclide / scintillation fluid.

675 ns (Fig. 8). On constate également une légère amélioration des limites de détections de 2,6\%. En comparant les facteurs de mérite à 75 ns et à 675 ns grâce au test En (Tab. 1), on n'observe pas de différence significative. En effet le En calculé est inférieur à 1,2, néanmoins la valeur reste élevée, montrant que l'optimisation du DBB a un impact sur le rapport efficacité/bruit de fond. Lorsque l'on compare les valeurs d'activités mesurées, bien que beaucoup plus proches de la valeur cible dans le second cas, il n'est pas observé de différence significative entre celles calculées à 75 ns et celles à 675 ns; le test En par rapport à la valeur du certificat donne respectivement des valeurs de 0,8 et 0,3 (Tab. 1), démontrant que quelle que soit la valeur du DBB le résultat d'activité rendu reste juste. Dans ce cas de préparations avec l'Ultima Gold LTT, bien que l'influence ne soit pas majeure, un réglage à 675 ns rend des résultats plus performants.

\subsubsection{Cas du ${ }^{89} \mathrm{Sr}(\operatorname{Emax}=1495,1 \mathrm{kev})$}

Sur les préparations réalisées avec du Pico-Fluor 40, aucune différence significative n'est observée quelle que soit la valeur du DBB (Tab. 1). Le signal mesuré provenant de la source n'indique pas de hausse sensible, et il en est de même pour la préparation correspondant à un blanc, se traduisant par un facteur de mérite identique. L'efficacité de comptage calculée est la même pour les deux valeurs de délai étudiées (Fig. 8) et les limites de détection obtenues sont équivalentes (Fig. 9). Lorsque l'on compare les valeurs d'activités mesurées, bien que beaucoup plus proches de la valeur cible dans le premier cas, il n'est pas observé de différence significative entre celles calculées à 75 ns et celles à 675 ns; le test En par rapport à la valeur du certificat donnant respectivement des valeurs de 0,2 et 0,9 (Tab. 1), ce qui démontre que quelle que soit la valeur du DBB le résultat d'activité rendu reste juste. En revanche, avec les préparations contenant de l'Ultima Gold LLT, il est constaté une différence significative entre les deux valeurs de DBB (Tab. 1). L'efficacité de comptage calculée est sensiblement améliorée, le facteur de mérite augmente et le test En largement supérieur à 1,2 montre que les résultats sont significativement différents. La limite de détection est diminuée de $16,8 \%$ (Tab. 1). Lorsque l'on compare les valeurs d'activités mesurées, il n'est pas observé de différence significative entre celles calculées à 75 ns et celles à 675 ns; le test En par rapport à la valeur du certificat donnant respectivement des valeurs de 0,1 et 0,2 (Tab. 1) ce qui démontre que le DBB n'a pas d'influence sur le résultat d'activité rendu. Dans ce cas de préparations avec l'Ultima Gold LTT, il est donc très important que ce réglage soit optimisé car l'impact sur le résultat global peut être important et non négligeable.

\subsubsection{Cas du ${ }^{90} \mathrm{Sr} /{ }^{90} \mathrm{Y}(\mathrm{Emax}=2279,8 \mathrm{kev})$}

Concernant les préparations réalisées avec du Picofluor 40, et ce pour les deux valeurs de DBB étudiées, le rapport efficacité sur bruit de fond reste le même (Fig. 7), les limites de détections sont identiques (Fig. 9). Les résultats du test En sur les activités mesurées montrent qu'elles sont justes par rapport à la valeur cible (Tab. 1). Dans ce cas, l'optimisation du paramètre n'a pas d'influence sur le résultat final.

En revanche, comme dans le cas du ${ }^{89} \mathrm{Sr}$, pour les préparations réalisées avec de l'Ultima Gold LLT, l'efficacité de comptage est nettement améliorée (Fig. 8). Le test En sur les facteurs de mérite avec une valeur largement supérieure à 1,2 indique une différence significative entre les deux résultats. La limite de détection est améliorée de 17,4\% (Tab. 1). Comme dans les autres cas, l'activité mesurée n'est pas différente de l'activité cible (Tab. 1). Au même titre que dans le cas du ${ }^{89} \mathrm{Sr}$, il est donc important que ce réglage soit optimisé lors de mesures avec l'Ultima Gold LLT.

\subsection{Discussion}

Les résultats montrent que quelle que soit l'énergie de l'émetteur $\beta$, faible, moyenne ou élevée, la valeur de DBB n'a pas d'influence lorsque la mesure est réalisée avec du PicoFluor 40. En revanche, à la valeur de 675 ns en utilisant l'Ultima Gold LLT, le rapport de l'efficacité sur le mouvement propre est meilleur, se traduisant par des facteurs de mérite relativement plus élevés (Fig. 7); ce phénomène est davantage 
observable sur les émetteurs $\beta$ de fortes énergies. Dans tous les cas, les limites de détection les plus basses sont obtenues avec l'Ultima Gold LLT.

Dans le cas du ${ }^{89} \mathrm{Sr}$ et du couple ${ }^{90} \mathrm{Sr} /{ }^{90} \mathrm{Y}$, émetteurs $\beta$ de forte énergie, bien que développé pour les émetteurs de faibles énergies, l'utilisation de l'Ultima Gold LLT semble être un bon choix quelle que soit la valeur du DBB. Les limites de détection sont plus performantes avec un DBB réglé à 675 ns.

De manière générale, l'optimisation du paramètre DBB n'a pas d'influence sur la justesse de l'activité mesurée : dans tous les cas étudiés nous avons pu observer que les valeurs de test En par rapport aux valeurs assignées étaient systématiquement inférieures à 1,2. En revanche, les limites de détections sont toutes améliorées lors de l'utilisation de l'Ultima Gold LLT.

\section{Conclusion et perspectives}

Ces dernières années ont vu apparaître de nouveaux liquides scintillants tels que l'Ultima Gold LLT, de qualité biodégradable, tandis que la production de liquides scintillants traditionnels, tels que le Pico-Fluor 40, était amenée à s'arrêter.

L'utilisation de cette nouvelle génération de liquides scintillants révèle un phénomène physique interférant sur la qualité de la mesure : la dissipation de l'énergie des photons dans le milieu est plus longue. Cette caractéristique des liquides scintillants de nouvelle génération contraint le laboratoire à réviser ses protocoles de mesures pour maintenir des efficacités de comptage performantes et atteindre des limites de détection aussi basses que possible.

L'influence du DBB a été analysée selon ces objectifs. Les résultats montrent que le choix de ce facteur est déterminant pour l'amélioration des performances de la mesure des radionucléides émetteurs $\beta$ notamment lorsqu'il s'agit d'émetteurs $\beta$ d'énergie élevée comme les strontium.

Lorsque la valeur du DBB est augmentée de 75 ns à 675 ns, les efficacités de comptage du ${ }^{14} \mathrm{C}$ et du tritium sont légèrement améliorées, alors que celles du ${ }^{89} \mathrm{Sr}$ et du couple ${ }^{90} \mathrm{Sr} /{ }^{90} \mathrm{Y}$ deviennent significativement meilleures.

L'optimisation du paramétrage permet d'abaisser les limites de détection, facteur favorable à une meilleure détection des radionucléides émetteurs $\beta$ présents dans les échantillons mesurés au laboratoire. Ces limites de détection pourraient être encore abaissées en sélectionnant les fenêtres de comptage spécifiques à chaque radionucléide, et en utilisant le mode bas bruit de fond des compteurs à scintillation liquide.

Théoriquement, la valeur de DBB devrait être optimisée pour chacun des radionucléides mesurés. Les résultats montrent qu'un réglage du DBB à des valeurs supérieures à $200 \mathrm{~ns}$ est satisfaisant pour les émetteurs $\beta$ de faibles et moyennes énergies. Pour les émetteurs $\beta$ énergétiques, $l^{90} \mathrm{Y}$ par exemple, le réglage du DBB est optimal à $675 \mathrm{~ns}$. Toutefois, en pratique, le laboratoire applique un protocole commun à la mesure directe de tous les émetteurs $\beta$. La valeur de 675 ns a donc été retenue, comme compromis, pour couvrir la gamme d'énergie la plus étendue possible qui corresponde aux radionucléides mesurés au laboratoire. Cette valeur est considérée optimale et permet d'atteindre des limites de détection raisonnablement basses pour chacun des radionucléides mesurés.

Cette étude ayant été menée en utilisant un volume de scintillant fixe, à l'avenir, le LAMR projette d'étudier l'influence du volume des scintillants sur les performances de la détection. L'un des objectifs est de réduire les volumes de déchets, et de déterminer le meilleur rapport volume échantillon/volume liquide scintillant.

Dans le cadre du maintien de l'accréditation COFRAC sur le dosage direct des émetteurs $\beta$, le laboratoire doit procéder à des mesures comparatives entre l'Ultima Gold LLT et le PicoFluor 40. Pour valider les performances du liquide scintillant nouvelle génération, trois niveaux d'énergies seront testés avec $\mathrm{du}{ }^{3} \mathrm{H}$, du ${ }^{14} \mathrm{C}$ et du ${ }^{89} \mathrm{Sr}$, trois niveaux d'activité (couvrant ainsi tout le domaine de mesure) et trois niveaux de quenching.

\section{Références}

Cassette Ph. (2004) Mesure de la radioactivité par scintillation liquide. Dans : Techniques de l'ingénieur. Référence P2552.

CEA/LNE (2007) Mini table de radionucléides. EDP sciences, Les Ulis.

G.T.N.5 (1989) Détermination du seuil et de la limite de détection en spectrométrie gamma. Groupe de travail de Normalisation $\mathrm{n}^{\circ} 5$. Rapport CEA-R-5506.

Horrocks D.L. (1974) Application of liquid scintillation counting, pp. 12-33.Academic Press, New York.

Passo C.J. Jr., Kessler M.J.(1992) Selectable Delay Before Burst - A novel feature to enhance low-level counting performance. Dans : Liquid Scintillation Spectrometry. (J.E. Noakes, F. Schönhofer, H.A. Polach, Eds) pp.51-57. Radiocarbon, Tucson.

Perkin Elmer (2007) Scintillation Cocktails and Consumables, pp. 6-12. Perkin Elmer Inc., Waltham.

Simonnet G. (1994) Les radioisotopes en recherche biologique Détection et radioprotection, pp. 43-94. Masson, Paris.

Tymen H., Robert N., Girard de Vasson O., Fottorino R. (1998) Méthode de dosage du technétium 99 dans les urines par scintillation liquide, Radioprotection 33, 147-158.

Cite this article as: G. Finance, J. Siscard, M. Agarande, D. Franck, P. Boisson. Optimisation de la mesure urinaire des émetteurs bêta par scintillation liquide à résolution temporelle. Radioprotection 49(1), 55-60 (2014). 